\title{
Vibrational states for hydrogen in palladium
}

\author{
C. Elsässer \\ Max-Planck-Institut für Metallforschung, Institut für Physik, Heisenbergstrasse 1, D-7000 Stuttgart 80, Germany \\ K. M. Ho and C. T. Chan \\ Ames Laboratory and Department of Physics, Iowa State University, Ames, Iowa 50011 \\ M. Fähnle \\ Max-Planck-Institut für Metallforschung, Institut für Physik, Heisenbergstrasse 1, D-7000 Stuttgart 80, Germany
}

(Received 21 June 1991)

\begin{abstract}
Vibrational potentials for hydrogen isotopes in $\mathrm{PdH}$ and $\mathrm{Pd}_{4} \mathrm{H}$ are determined from first-principles total-energy calculations. The strong anharmonicity of the potential for $\mathrm{H}$ around octahedral sites must be taken into account beyond perturbation theory. For PdH good quantitative agreement of our calculated vibration energies with inelastic-neutron-scattering (INS) results is achieved, allowing a reinterpretation of the INS spectra. For lower $\mathrm{H}$ concentrations $\left(\operatorname{Pd}_{n} \mathrm{H}, n=4,8,16\right.$, and 32) the influence of local lattice relaxations on the vibration potentials and energies is important. Furthermore, metastable states confined to tetrahedral sites in $\mathrm{Pd}$ are found for the heavier isotopes ${ }^{2} \mathrm{D}$ and ${ }^{3} \mathrm{~T}$.
\end{abstract}

The investigation of hydrogen isotopes in metals has led to a detailed understanding of the interaction between the metal host lattice and the light interstitials. ${ }^{\prime}$ In this paper we present a theoretical study of the vibrational states of $\mathrm{H}$ in $\mathrm{PdH}_{x}$ (Ref. 2) ( $x$ is the hydrogen concentration). These vibrations are of fundamental importance in the study of superconductivity ${ }^{3}$ and hydrogen diffusion ${ }^{4}$ in such systems.

Experimentally, the vibrational properties of interstitial $\mathrm{H}$ in Pd (Ref. 5) single crystals were studied with inelastic neutron scattering (INS). ${ }^{6}$ The measured excitation spectra were commonly interpreted in terms of oscillator states in a three-dimensional potential well. ${ }^{7}$ Anharmonicity was sometimes taken into account using perturbation theory. ${ }^{5}$ Theoretically, vibration energies and wave functions of $\mathbf{H}$ in body-centered-cubic (bcc) and facecentered-cubic (fcc) metals were calculated using empiri$\mathrm{cal}^{8}$ or semiempirical ${ }^{9}$ potential models. However, it is not clear that such models provide an accurate description of the metal-hydrogen interaction.

Some years ago the vibration potential and excitation energies for $\mathrm{H}$ in the $\gamma$ phase of $\mathrm{NbH}$ were calculated from first principles, ${ }^{10}$ and the results were in quantitative agreement with the available INS data. Motivated by the success of this work, we applied similar techniques to study the more complicated case of $\mathrm{H}$ in $\mathrm{PdH}$ and $\mathrm{PdH}_{0.25}$. These two systems are chosen to represent two coexisting phases in real $\mathrm{PdH}_{x}$ samples: In $\beta$-phase regions $\mathrm{H}$ and $\mathrm{Pd}$ atoms form a rocksalt structure. In $\alpha$ phase regions few $\mathrm{H}$ atoms are distributed over the Pd lattice.

As in the previous work, ${ }^{10}$ the calculations are performed using the pseudopotential approach within the local-density-functional approximation 11 (LDA) and the Born-Oppenheimer approximation (BOA). The atomic cores are represented by a nonlocal, norm-conserving ionic pseudopotential ${ }^{12}$ for $\mathrm{Pd}^{10+}$ and a local norm-conserving pseudopotential for $\mathrm{H}^{+}$. The latter is constructed from the analytic $1 s$ state of the hydrogen problem via Kerker's scheme, ${ }^{13}$ which was modified to yield a smooth potential in real space. The electronic wave functions and charge densities are represented by a mixed basis ${ }^{14}$ of plane waves $\left(|\mathbf{k}+\mathbf{G}|^{2} \leq 16.5 \mathrm{Ry}\right)$ and localized numerical functions centered at the atomic sites. For each Pd atom five $d$ functions are used, for each $\mathrm{H}$ atom one $s$ function. Total energies are calculated in the LDA using a momentumspace formalism. ${ }^{15}$

For $\mathrm{PdH}$ a fcc unit cell with one $\mathrm{Pd}$ and one $\mathrm{H}$ atom was used. The calculated equilibrium lattice constant of the unit cell with $\mathrm{H}$ at the octahedral site is $a_{0}=4.07 \AA$, which is close to $a_{\text {expt }}=4.09 \AA$ for $\beta-\mathrm{PdH}_{1}{ }^{2} \mathrm{PdH}_{0.25}$ is modeled by a simple cubic (sc) unit-cell $\mathrm{Pd}_{4} \mathrm{H}$ with four $\mathrm{Pd}$ atoms forming a fec lattice and one $\mathrm{H}$ atom. For $\mathrm{H}$ at an octahedral site the calculated equilibrium lattice constant is $a_{0}=3.94 \AA$, in comparison to $a_{\text {expt }}=3.95 \AA$ for a $\mathrm{PdH}_{0.25}$ sample. ${ }^{2}$ For both unit cells the $\mathrm{H}$ atom is shifted along several directions around the stable octahedral site and the metastable tetrahedral site (see Fig. 1) and total energies are calculated to map out the adiabatic interaction potentials. The energy versus displacement curves for $\mathrm{PdH}$ and $\mathrm{Pd}_{4} \mathrm{H}$ are shown in Fig. 2.

For the vibrational states of hydrogen, we made the BOA once again for the heavy metal atoms and the light hydrogen isotopes. Hydrogen is considered to vibrate in a stiff metal lattice. Vibration states are determined by solution of the Schrödinger equation of a particle moving in a three-dimensional potential. The potential in the BOA is represented by fits of analytical functions $V(x, y, z)$ to the total-energy data $(x, y, z$ are Cartesian coordinates). We proceeded by applying two different kinds of functions $V(x, y, z)$.

In the first step we followed the suggestions given in the literature ${ }^{5}$ for the interpretation of INS spectra via polynomial potential wells which are centered at the local potential minima and have the correct point symmetries. Up to fourth order in the Cartesian coordinates, for an octa- 


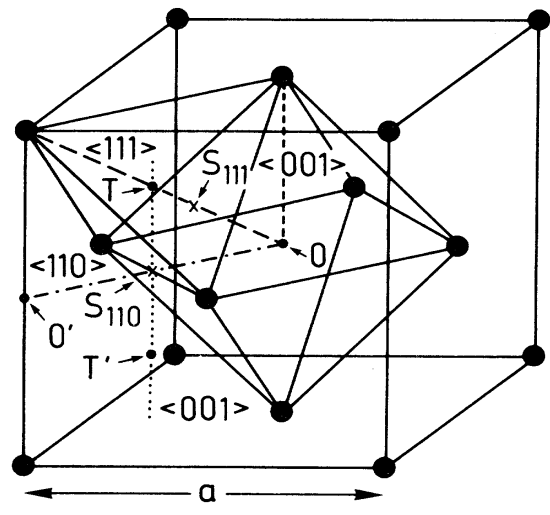

FIG. 1. Interstice of the fcc Pd lattice; $O, O^{\prime}$ : octahedral sites; $T, T^{\prime}$ : tetrahedral sites; $S_{110}$ : middle of dumbbell; $S_{111}$ : middle of triangle; $\langle i j k\rangle$ : directions in the cubic lattice.

hedral and a tetrahedral site in $\mathrm{Pd}$ the potential $V(x, y, z)$ is given by ${ }^{5}$

$$
\begin{aligned}
V(x, y, z)= & c_{2}\left(x^{2}+y^{2}+z^{2}\right)+c_{3} x y z+c_{4}\left(x^{4}+y^{4}+z^{4}\right) \\
& +c_{22}\left(x^{2} y^{2}+y^{2} z^{2}+z^{2} x^{2}\right),
\end{aligned}
$$

with $c_{3}=0$ for the octahedral site. The coefficients were determined by least-squares fits to 9 and 7 data points surrounding an octahedral or tetrahedral site in $\mathrm{PdH}$ and
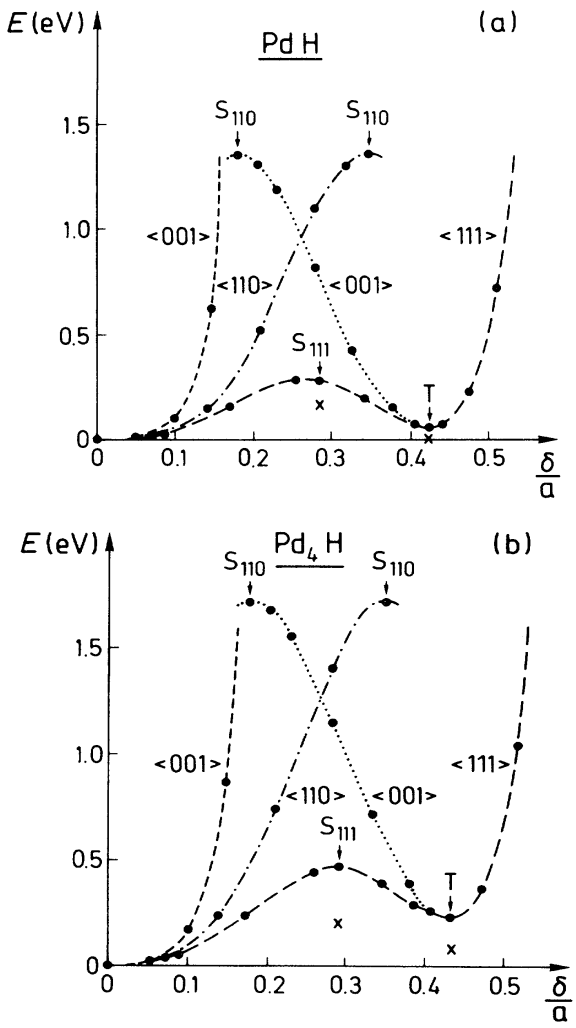

FIG. 2. Energy vs displacement curves for $\mathbf{H}$ in $\mathrm{PdH}$ [(a) $a=4.07 \AA$ ] and $\mathrm{Pd}_{4} \mathrm{H}$ [(b) $a=3.94 \AA$ ]; origin: octahedral minimum; $T$ : tetrahedral minimum; $S_{110}$ and $S_{111}$ : saddle points; $\bullet$ : ab initio data, $\times$ : data including lattice relaxations.
$\mathrm{Pd}_{4} \mathrm{H}$, respectively. Corrections to the eigenstates of a three-dimensional harmonic oscillator (second-order potential terms) due to the anharmonicity of the potentials were calculated using standard perturbation theory. ${ }^{16}$ The fourth-order potential terms lead to first-order perturbation corrections, the third-order terms to secondorder corrections.

As a second type of potential function a finite, threedimensional Fourier series was fitted to energy versus displacement data throughout the whole unit cell: ${ }^{10}$

$$
V(\mathbf{r})=\sum_{\mathbf{G}} V_{\mathbf{G}} e^{i \mathbf{G} \cdot \mathbf{r}} .
$$

In contrast to the local potential wells, this function takes both the translational periodicity of the potential and the saddle points between the different local minima into account. For $\mathrm{PdH}\left(\mathrm{Pd}_{4} \mathrm{H}\right)$ we fitted the Fourier components of 19 (12) independent stars of reciprocal lattice vectors $\mathbf{G}$ to the energies of all 30 (29) different $\mathrm{H}$ positions shown in Fig. 2. Then the three-dimensional Schrödinger equation for $\mathrm{H}$ isotopes in the periodic potential was expanded in plane waves and the resulting matrix was diagonalized for the $\Gamma$ point as described in Ref. 10 for $\mathrm{NbH}$. In this approach anharmonicity is treated completely, not as a perturbation.

Our calculated excitation energies, $e_{n m l}=E_{n m l}-E_{000}$ $(n, m, l=0,1,2, \ldots$, are quantum numbers of the corresponding harmonic-oscillator states), for the three $\mathrm{H}$ isotopes are compiled in Tables I-III, and compared with experimental data. ${ }^{5}$

For vibrations of $\mathrm{H}$ around the octahedral site in volume-relaxed $\mathrm{PdH}$ and $\mathrm{Pd}_{4} \mathrm{H}$, the energies calculated with the polynomial potential do not agree with measured

TABLE I. Calculated and measured excitation energies (degeneracies in superscripts) of vibrations of $\mathrm{H}$ isotopes in octahedral sites of $\mathrm{PdH}$ (in $\mathrm{meV}$ ); (a) harmonic approximation, (b) perturbation theory, and (c) solution of Fourier representation. The experimental data are taken from Ref. 5, as discussed in the

\begin{tabular}{|c|c|c|c|c|}
\hline$e_{n m ! l}^{(d)}$ & & ${ }^{1} \mathrm{H}$ & ${ }^{2} \mathrm{D}$ & ${ }^{3} \mathrm{~T}$ \\
\hline \multirow[t]{3}{*}{$e_{100}^{(3)}$} & (a) & 34 & 23 & 20 \\
\hline & (b) & 126 & 70 & 50 \\
\hline & (c) & 62 & 40 & 32 \\
\hline \multirow[t]{2}{*}{ expt. } & $\beta$ & 60 & 40 & \\
\hline & $\alpha$ & $69.0 \pm 0.5$ & $46.5 \pm 0.5$ & \\
\hline \multirow[t]{3}{*}{$e_{110}^{(3)}$} & (a) & 68 & 47 & 39 \\
\hline & (b) & 226 & 127 & 92 \\
\hline & (c) & 117 & 78 & 61 \\
\hline expt. & $\alpha$ & $115 \pm 5$ & & \\
\hline \multirow[t]{3}{*}{$e_{200}^{(1)}$} & (a) & 68 & 47 & 39 \\
\hline & (b) & 344 & 186 & 131 \\
\hline & (c) & 132 & 88 & 69 \\
\hline expt. & $\alpha$ & $137 \pm 2$ & & \\
\hline \multirow[t]{3}{*}{$e_{200}^{(2)}$} & (a) & 68 & 47 & 39 \\
\hline & (b) & 383 & 206 & 144 \\
\hline & (c) & 147 & 94 & 73 \\
\hline expt. & $\alpha$ & $156 \pm 3$ & & \\
\hline
\end{tabular}
text ( $\alpha$ and $\beta$ denote the dominant phase of the samples $\mathrm{PdH}_{0.014}$ and $\mathrm{PdH}_{0.63}$, respectively). 
TABLE II. Calculated and measured excitation energies (degeneracies in superscripts) of vibrations of $\mathrm{H}$ isotopes in octahedral sites of $\mathrm{Pd}_{4} \mathrm{H}$ (in meV); for the meaning of (a)-(c) see caption of Table I.

\begin{tabular}{lcccc}
\hline \hline$e_{n m l}^{(d)}$ & & ${ }^{1} \mathrm{H}$ & ${ }^{2} \mathrm{D}$ & ${ }^{3} \mathrm{~T}$ \\
\hline$e_{100}^{(3)}$ & $(\mathrm{a})$ & 53 & 38 & 31 \\
& $(\mathrm{~b})$ & 113 & 68 & 51 \\
& $(\mathrm{c})$ & 83 & 54 & 43 \\
expt. & $\beta$ & 60 & 40 & \\
& $\alpha$ & $69.0 \pm 0.5$ & $46.5 \pm 0.5$ & \\
$e_{110}^{(3)}$ & $(\mathrm{a})$ & 107 & 76 & 62 \\
& $(\mathrm{~b})$ & 206 & 125 & 95 \\
& $(\mathrm{c})$ & 158 & 104 & 82 \\
expt. & $\alpha$ & $115 \pm 5$ & & \\
$e_{200}^{(1)}$ & $(\mathrm{a})$ & 107 & 76 & 62 \\
& $(\mathrm{~b})$ & 286 & 165 & 121 \\
& $(\mathrm{c})$ & 179 & 118 & 93 \\
expt. & $\alpha$ & $137 \pm 2$ & & \\
$e_{200}^{(2)}$ & $(\mathrm{a})$ & 107 & 76 & 62 \\
& $(\mathrm{~b})$ & 317 & 180 & 132 \\
& $(\mathrm{c})$ & 197 & 128 & 100 \\
expt. & $\alpha$ & $156 \pm 3$ & & \\
\hline \hline
\end{tabular}

INS data, as shown in Tables I and II. The harmonic approximation (a) yields energies which are significantly too low. By taking the anharmonicity into account via perturbation theory (b) the energies become much too high. The origin for these discrepancies is the strong anisotropy and anharmonicity of the adiabatic potential, which cannot be adequately described by a local polynomial function and perturbation theory. In contrast, for $\mathbf{P d H}$, the vibration energies calculated with the Fourier potential (c), which is not confined to the local surrounding of an octahedral site, agree much better with the available INS data. For $\mathbf{P d}_{4} \mathrm{H}$, the calculated energies are still somewhat too large compared with experiment, as may be expected from the steeper adiabatic potential (see Fig. 2). But this discrepancy can be related to the neglect of local relaxations of the Pd atoms, as shown below. The principal conclusion for $\mathrm{H}$ on octahedral sites in $\mathrm{Pd}$ is that the strong anharmonicity and anisotropy of the potential requires a calculation of vibrational states beyond harmonic or perturbative approximations.

TABLE III. Calculated zero-point and first-excitation energies (degeneracies in superscripts) of vibrations of $\mathrm{H}$ isotopes in tetrahedral sites of $\mathrm{PdH}$ (in $\mathrm{meV}$ ); for the meaning of (a)-(c) see caption of Table I.

\begin{tabular}{llccc}
\hline \hline & & ${ }^{1} \mathrm{H}$ & ${ }^{2} \mathrm{D}$ & ${ }^{3} \mathrm{~T}$ \\
\hline$E_{000}^{(1)}$ & (a) & 204 & 144 & 117 \\
& (b) & 208 & 146 & 119 \\
& (c) & 213 & 150 & 123 \\
$e_{100}^{(3)}$ & (a) & 136 & 96 & 78 \\
& (b) & 124 & 90 & 75 \\
& (c) & & 92 & 77 \\
\hline \hline
\end{tabular}

When we compare the calculated and measured energies, we need to note the following. First, in the INS experiments ${ }^{5}$ the higher excitation energies were measured in $\alpha$-phase samples $\mathrm{PdH}_{0.014}$, whereas the $\mathrm{PdH}$ unit cell used in our calculations corresponds to the $\beta$ phase. Besides $\mathrm{Pd}_{4} \mathrm{H}$ we did calculations with further $\mathrm{Pd}_{n} \mathrm{H}$ supercells $(n=8,16,32)$ to approach the $\alpha$ phase. ${ }^{17}$ The decreasing $\mathrm{H}$ concentration leads to a reduction of the equilibrium lattice constant of the ideal fcc lattice. This is connected with a steeper vibration potential and higher vibration energies (see Fig. 4 in Ref. 17). So far no relaxations of the unit-cell volume and the positions of Pd atoms for arbitrary $\mathbf{H}$ positions were taken into account, which can modify the adiabatic potentials quantitatively. ${ }^{17} \mathrm{We}$ studied the influence of relaxations for $\mathbf{H}$ at a tetrahedral site $T$ and a saddle point $S_{111}$ (see Fig. 3 of Ref. 17). In $\mathrm{PdH}$ unit cells only the relaxation of lattice constants is possible. In $\mathrm{Pd}_{4} \mathrm{H}$ and $\mathrm{Pd}_{8} \mathrm{H}$ local lattice deformations around the interstitial $H$ are found, which increase the nearest-neighbor $\mathrm{Pd}-\mathrm{H}$ distances and reduce the steepness of the adiabatic potentials. This explains the overestimation of the calculated vibration energies for the unrelaxed $\mathrm{Pd}_{4} \mathrm{H}$. They would become smaller and agree better with experiment if an adiabatic potential for fully relaxed $\mathrm{Pd}_{4} \mathrm{H}$ were available. An ab initio determination of such a potential would be a very time-consuming task. Therefore, in a subsequent project, we are working on a connection of our ab initio study with an empirical model scheme mentioned above. ${ }^{8}$

Second, in the interpretation of their INS spectra, the authors of Ref. 5 did not consider the splitting of the sixfold degenerate harmonic level $E_{200}^{h}=E_{110}^{h}$ into a threefold degenerate $E_{110}^{(3)}$, a twofold $E_{200}^{(2)}$, and a single level

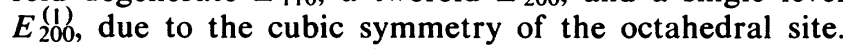
Besides the large peak from the first excitation, $e_{100}$, in the INS spectra (see Fig. 1 in Ref. 5) they identified two further structures as threefold degenerate excitations $e_{110}$ and $e_{200}$. To compare our calculated vibration energies with the INS spectra in Ref. 5, we propose the following interpretation (see Table II): The two structures at $137 \pm 2 \mathrm{meV}$ and $156 \pm 3 \mathrm{meV}$ seem to be the excitations $e_{200}$ and $e_{200}$, respectively. The remaining $e_{110}^{(3)}$ excitation might be located around $115 \pm 5 \mathrm{meV}$ where the noise in the spectra does not obviously exclude a third small structure.

Besides the $\mathrm{H}$ vibrations around the stable octahedral site in $\mathrm{PdH}$ we also investigated the possibility for metastable $\mathrm{H}$ states confined to tetrahedral sites. Using the polynomial potential, zero-point and first excitation energies were calculated in the harmonic approximation and with perturbation theory. In Table III they are listed, together with energies found with the Fourier potential, which can be associated to tetrahedral sites via symmetry and convergence arguments. The energies are referred to the tetrahedral minimum, which is $51 \mathrm{meV}$ above the octahedral minimum (see Fig. 2). The results show that the local potential around a tetrahedral site is only weakly anharmonic. Metastable bound states at tetrahedral sites are expected at least if the zero-point energies are below the depth of the local potential well, which is limited by the difference of $215 \mathrm{meV}$ between the minimum at $T$ and 
the saddle-point energy at $S_{111}$. This implies that an occupation of a tetrahedral site is more likely for ${ }^{3} \mathrm{~T}$ or ${ }^{2} \mathrm{D}$ than for ${ }^{1} \mathrm{H}$.

The occupations of interstitial sites in fcc Pd were investigated by means of ion channeling experiments. ${ }^{18}$ For $\mathbf{P d H}_{x}$ and $\mathrm{PdD}_{x}$ at and above room temperatures, where the thermal vibration amplitudes of the interstitials around octahedral sites are already rather large, no evidences were found for occupations of tetrahedral sites. According to our calculations, we would expect to find a channeling signal along crystalline $\langle 100\rangle$ directions originating from tetrahedral-site occupations for the isotope ${ }^{3} \mathrm{~T}$ and eventually ${ }^{2} \mathrm{D}$ at low temperatures, where the $\langle 100\rangle$ blocking originating from octahedral-site occupation is less smeared out by the thermal vibrations. An occupation of tetrahedral sites by ${ }^{3} \mathrm{~T}$ would also be compatible with the observed anomalous isotope effect for activation energies of $\mathrm{H}$ diffusion in $\mathbf{P d} .^{4}$

The Ames Laboratory is operated for the U.S. Department of Energy by the Iowa State University under Contract No. W-7405-Eng-82. The work was supported in part by the Director for Energy Research, Office of Basic Energy Sciences, including a grant of computer time on the Cray computers at Lawrence Livermore Laboratory.
'Reviews are given in Hydrogen in Metals I, edited by G. Alefeld and J. Völkl, Topics in Applied Physics Vol. 28 (Springer, Berlin, 1978); Hydrogen in Metals II, edited by G. Alefeld and J. Völkl, Topics in Applied Physics Vol. 29 (Springer, Berlin, 1978); Hydrogen in Intermetallic Compounds I, edited by Franz L. Schlapbach, Topics in Applied Physics Vol. 63 (Springer, Berlin, 1988); Sputtering by Particle Bombardment III, edited by $\mathrm{R}$. Behrisch and $\mathrm{K}$. Wittmaack, Topics in Applied Physics Vol. 64 (Springer, Berlin, 1988).

${ }^{2}$ E. Wicke, H. Brodowsky, and H. Züchner, Hydrogen in Metals II (Ref. 1), p. 73.

${ }^{3}$ D. A. Papaconstantopoulos, B. M. Klein, E. N. Economou, and L. L. Boyer, Phys. Rev. B 17, 141 (1978).

${ }^{4}$ H. Teichler, Z. Phys. Chem. 114, 155 (1979); H. Kronmüller, G. Higelin, P. Vargas, and R. Lässer, ibid. 143, 161 (1985).

5J. J. Rush, J. M. Rowe, and D. Richter, Z. Phys. B 55, 283 (1984).

${ }^{6} \mathrm{~T}$. Springer, Hydrogen in Metals I (Ref. 1), p. 75.

${ }^{7}$ H. R. Schober and V. Lottner, Z. Phys. Chem. 114, 203 (1979).

${ }^{8}$ H. Sugimoto and Y. Fukai, J. Phys. Soc. Jpn. 51, 2554 (1982); A. Klamt and H. Teichler, Phys. Status Solidi (b) 134, 103 (1986); 134, 533 (1986).
${ }^{9}$ M. J. Puska and R. M. Nieminen, Phys. Rev. B 29, 5382 (1984).

${ }^{10}$ K.-M. Ho, H.-J. Tao, and X.-Y. Zhu, Phys. Rev. Lett. 53, 1586 (1984); H.-J. Tao, K.-M. Ho, and X.-Y. Zhu, Phys. Rev. B 34, 8394 (1986).

${ }^{1}$ For recent reviews, see, e.g., R. O. Jones and O. Gunnarsson, Rev. Mod. Phys. 61, 689 (1989); W. E. Pickett, Comput. Phys. Rep. 9, 115 (1989).

${ }^{12}$ D. R. Hamann, M. Schlüter, and C. Chiang, Phys. Rev. Lett. 43, 1494 (1979); G. B. Bachelet and M. Schlüter, Phys. Rev. B 25, 2103 (1982).

${ }^{13}$ G. P. Kerker, J. Phys. C 13, L189 (1980).

${ }^{14}$ S. G. Louie, K. M. Ho, and M. L. Cohen, Phys. Rev. B 19, 1774 (1979); C. Elsässer, N. Takeuchi, K. M. Ho, C. T. Chan, P. Braun, and M. Fähnle, J. Phys. Condens. Matter 2, 4371 (1990).

15 J. Ihm, A. Zunger, and M. L. Cohen, J. Phys. C 12, 4409 (1979); 13, 3095 (1980).

${ }^{16}$ See textbooks about quantum mechanics, for instance, D. I. Blodninzew, Grundlager der Quantenmednaník (Thun, Harri Deutsch, 1985), in german.

${ }^{17}$ C. Elsässer, M. Fähnle, K. M. Ho, and C. T. Chan, Physica B 172, 217 (1991); and (unpublished).

${ }^{18}$ H. D. Carstanjen, Z. Phys. Chem. 165, 141 (1989). 